\title{
Polarimetric Evidence of Non-spherical Winds
}

\author{
Antônio Mário Magalhães ${ }^{1}$ and Cláudia V. Rodrigues ${ }^{2}$ \\ 1 IAG, Univ. São Paulo, Caixa Postal 3386 - São Paulo SP 01060-970 - Brazil \\ 2 INPE, Av. dos Astronautas, 1758 - São José dos Campos SP 12227-900 - Brazil
}

\begin{abstract}
Polarization observations yield otherwise unobtainable information about the geometrical structure of unresolved objects. In this talk we review the evidences for non-spherically symmetric structures around Luminous Hot Stars from polarimetry and what we can learn with this technique. Polarimetry has added a new dimension to the study of the envelopes of Luminous Blue Variables, WolfRayet stars and $\mathrm{B}[\mathrm{e}]$ stars, all of which are discussed in some detail.
\end{abstract}

\section{Introduction}

In the past few years there has been mounting evidence that the mass loss in Luminous Hot Stars (LHS) is non-spherically symmetric and this meeting is in fact a testimony to that. In addition, the abundance of free electrons in the winds of such objects makes Thomson scattering an important opacity source. This combination of asymmetry and scattered (hence polarized) light may result in an observed degree of polarization in the radiation we detect from LHS. Polarization observations carry then great potential to explore the environment of LHS.

In this talk we review the evidences for non-spherically symmetric structures around LHS from polarimetry and what we can learn from such data about the physics of such structures. Recent related reviews include those of Bjorkman (1994) and Schulte-Ladbeck (1997). Several talks in this conference also have direct bearing on the topic (K. Bjorkman, Brown and Ignace, Eversberg et al., Rodrigues and Magalhães, Schulte-Ladbeck et al.).

\section{Some Polarimetry Basics}

One great asset of polarization observations is that they yield diagnostics related to the geometrical structure of unresolvable objects. Generally, it can be said that the polarization is the ratio between the scattered flux and the total flux from the object. The polarization from a stellar envelope will depend in detail on the density and geometrical distribution of matter around the star (e.g., Wood et al. 1996). Techniques for measuring polarization in the UV-optical-IR have greatly advanced in recent years (Roberge and Whittet 1996; Magalhães et al. 1996).

The polarimetric wavelength dependence may be modified by any competing opacity and any unpolarized, diluting light from the star and/or wind. 
Examples include hydrogen bound-free and free-free opacities as well as line opacity such as from iron. Hydrogen recombination line emission tends to decrease the polarization across corresponding features, such as Balmer lines. All this provides valuable wind diagnostics. Dust scattering can also play a role in the outskirts of evolved objects. The wavelength dependence of single dust scattering depends on the nature of the grains and their size.

While this review will be concerned mostly with linear polarization, circular polarization may also in principle arise from processes such as multiple dust scattering in an envelope or magneto-emission from stellar spots. Electron scattering produces no circular polarization by itself.

Intrinsic polarization may be detected from the time variability of the observed polarization. In addition, the scatter of the data points in the Q-U diagram $(\mathrm{Q}=\mathrm{P} \cdot \cos (2 \theta)$ and $\mathrm{U}=\mathrm{P} \cdot \sin (2 \theta)$, where $\mathrm{P}=$ percent polarization, $\theta=$ position angle) will tell whether there is a preferred plane of symmetry or not. Binary stars where the scattering envelope surrounds one of them will show up as loops in the Q-U diagram (Brown et al. 1978). Intrinsic polarization may also show up through spectropolarimetry. If the observed polarization varies across a line, such as $\mathrm{H} \alpha$, the vector difference in the $\mathrm{Q}-\mathrm{U}$ plane of the continuum and line polarizations will provide the position angle (PA) of the intrinsic polarization (e.g., Schulte-Ladbeck et al. 1992).

\section{Observations of Luminous Hot Stars}

\subsection{Luminous Blue Variables}

Luminous Blue Variables (LBV) represent an intermediate stage between OB and WR stars (Maeder 1996). Direct evidence for asymmetric outflows comes from imaging (cf. Nota, these proceedings). In this case, spectropolarimetry has been used to probe mass loss on small spatial scales.

The $\mathbf{P}$ Cyg nebula has been resolved by direct imaging by Leitherer and Zickgraf (1987). P Cyg shows stochastic changes in its optical linear polarization (Hayes 1985), with night to night changes of $0.2 \%$ and $6^{\circ}$ in the polarization degree and PA, respectively.

Taylor et al. (1991a) have obtained spectropolarimetry of P Cyg for 20 nights during the 1989-1990 season. The observed polarization showed no preferred plane, consistent with random ejections of matter from the star. No correlation between increased line emission and polarization was observed. This was interpreted as a result from the time lag between these events, since about $40^{d}$ are required for a mass ejection to travel out to a distance of about $3 \mathrm{R}_{*}$, within which the polarization is thought to be produced.

Further constraints on P Cygni's envelope came from UV spectropolarimetry with WUPPE (Taylor et al. 1991b). A broad dip in the polarization around 2600-3000 $\AA$ suggested the existence of an absorptive opacity by FeII lines in the envelope. High resolution imaging of P Cyg (Nota et al. 1995; 


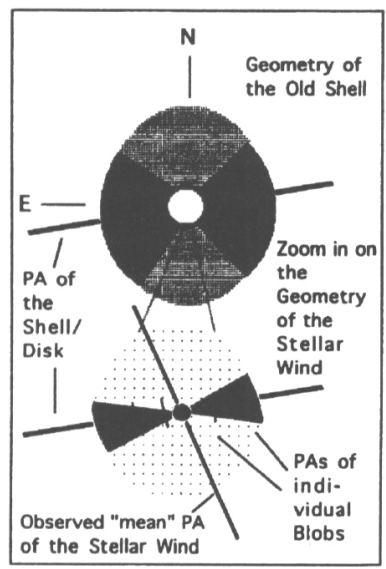

Fig. 1. Geometry of circumstellar wind of R127 (Schulte-Ladbeck et al. 1993).

Nota, these proceedings) shows that the structure of the envelope is indeed clumpy, nicely consistent with the structures seen much closer to the star in the polarimetry data.

The Large Magellanic Cloud LBV R 127 has been observed for spectropolarimetry by Schulte-Ladbeck et al. (1993). The intrinsic polarization, indicated by the line effect at $\mathrm{H} \alpha$, showed a level around $1.5 \%$ and was suggestive of electron scattering with possible FeII depression from within the envelope. The polarization was variable but with PA values restricted within a 'cone', with the interstellar value as apex, in the Q-U diagram.

The observed nebula (Clampin et al. 1993) is about $2 \mathrm{pc}$ in size and $\approx 10^{4}$ yr old. There are symmetric enhancements in the (coronographic) image along a direction $\approx 90^{\circ}$ from the polarization PA value. The suggested geometry for R127 (Schulte-Ladbeck et al. 1993; Fig. 1) is then that of a mass ejection in a preferred plane. The present geometry (from imaging) is defined by events taking place very close to the star (from polarimetry).

A few other LBV have been observed polarimetrically. In AG Car (Leitherer et al. 1994; Schulte-Ladbeck et al. 1994b), the geometry of the nebula shows an alignment with the PA derived from spectropolarimetry, with broad, polarized wings across $\mathrm{H} \alpha$ suggesting electron scattering. In HR Car (Clampin et al. 1995) the PA from imaging and that from polarimetry are actually the same, about $30^{\circ}$. However, we note that, according to Weis et al. (1997), the bipolar nebula has actually its axis at $\mathrm{PA} \approx 125^{\circ}$ and the imaging and polarimetry data are again consistent. Further monitoring of these objects to confirm the ejections in a preferred plane would be highly desirable.

In summary, polarimetry indicates that LBV may show either stochastic ejections (P Cyg) or, more commonly, a preferred plane for mass loss. In any 
case, the geometry present in the observed nebulae is already present in (and presumably imposed by) the wind very close to the star. Possible sources for this density contrast have been conjectured by Nota et al. (1995) but it is not possible yet to discern among them.

\subsection{Wolf-Rayet Stars}

Wolf-Rayet (WR) stars are the polarimetrically best studied class among the LHS (e.g., Robert et al. 1989; Moffat and Robert 1991; Drissen et al. 1992). For (presumed) single WR stars, there is a range in the observed variations of optical linear polarization: (a) WN stars vary more than WC ones in a given subclass; (b) Cooler sub-types (i.e., slower winds) vary more, although a few $(\approx 20 \%)$ WR show no variability; (c) Polarization variations have time scale of days and are wavelength independent; (d) Most WR show no preferred plane, but there are a few exceptions. Intraday variability is still poorly known.

For binary WR stars, cyclic variations of polarization with binary phase are often seen. This is due to the O-star light scattered off the dense WR wind. Mass loss rates can be derived (St.-Louis et al. 1988) as well as the inclination of the systems (Brown et al. 1978), providing important information about WR masses.

Circular polarimetry has been looked for in EZ Cma (Robert et al. 1992) with negative results, suggesting that the star does not show activity related to strong magnetic fields.

Harries et al. (1998) performed a spectropolarimetric survey of 16 WR. Their data are consistent with a distribution of intrinsic polarizations biased towards small values, with only $\approx 20 \%$ of stars with $\mathrm{P} \geq 0.3 \%$. Radiative transfer models suggest equator-to-pole density contrast of 2-3. Combining their results with literature data, for a total of 29 stars, the 5 known objects with 'line effects' cluster around the high mass loss \& luminosity part of the $\dot{M}$-L diagram (Fig. 2). Also, the $\dot{M}$ values from radio and optical are in good agreement, suggesting that the wind structures have density contrast independent of radius.

The results of Harries et al. (1998) seem to suggest that the global wind asymmetries in WR winds arise only in the fastest rotators (Ignace et al. 1996). Specially in view of the distribution of 'line effect' stars in the $\dot{M}-\mathrm{L}$ diagram, we feel that this is also supported by the fact that rotating stars evolve towards higher luminosity (Fliegner et al. 1996).

As the $\mathrm{O}$ component screens the WR envelope in an eclipse, the observed polarization may change dramatically and it can be used to model the WR wind (e.g., St-Louis et al. 1993; Rodrigues and Magalhães 1995). Spectropolarimetry across eclipses holds also great potential for probing the ionization structure of the wind. 


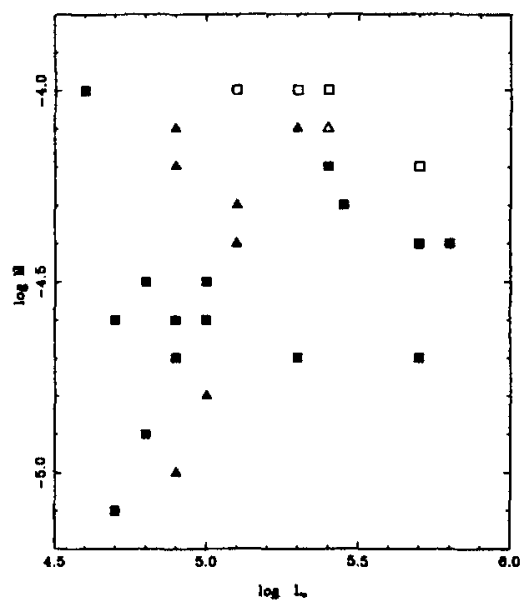

Fig. 2. Spherical (filled symbols) and non-spherical (open symbols) WR stars in the $\dot{M}$-L diagram (Harries et al. 1998).

\subsection{B $B$ e] Supergiants}

These objects show evidence of a two-component wind: a hot, fast polar wind and a denser, slow equatorial wind (see Zickgraf, these proceedings; de Araújo et al. 1994). Magalhães (1992) showed that the Magellanic B[e] supergiants do present intrinsic polarization, lending further support to the model put forward by Zickgraf et al. In addition, the higher intrinsic polarization values were all associated with objects spectroscopically found to be edge-on. The polarization of these systems, $P_{\text {edge-on }}$, correlated some with the average electron density $N_{e}$ of the envelopes but it correlated somewhat better with the IR [K-L] dust excesses.

Magalhães (1992) pointed out however that in the IR we tend to detect the larger grains, which are poor scatterers in the optical and might not polarize; instead, electrons closer to the star might be operative. Interestingly, the $P_{\text {edge-on }}-N_{e}$ correlation of Magalhães (1992) with the AV16/R4 (a binary, Zickgraf et al. 1996b) point removed becomes actually the tightest one. Spectropolarimetry of the most highly polarized object (S22, SchulteLadbeck and Clayton 1993) showed that electron scattering is indeed present, at least for that object. Monte Carlo scattering models (Melgarejo et al. 1999) suggest that homogeneous disks fit the polarization data for $\mathrm{B}[\mathrm{e}]$ stars well. This is consistent with the very slow winds observed and modeled by Zickgraf et al. (1996a) from spectroscopic data, providing another interesting link between the different types of observations. 
Three Magellanic B $[\mathrm{e}]$ stars have shown variability in polarization (S22, Schulte-Ladbeck and Clayton 1993; S18, Schulte-Ladbeck et al. 1994a) and photometry and spectroscopy (R4, Zickgraf et al. 1996b) similarly to LBV stars. While further scrutiny may show others to be variable too, Gayley \& Owocki 1995 have shown that the $\mathrm{B}[\mathrm{e}]$ class actually extends to luminosities much lower $\left(\log \mathrm{L} / \mathrm{L}_{\odot} \approx 4\right)$ than their supergiant counterparts $\left(\log \mathrm{L} / \mathrm{L}_{\odot} \approx\right.$ $5.5-6.0)$.

\subsection{Other Objects}

Lupie and Nordsieck (1987) showed that OB stars have intrinsic polarization. An on-going spectropolarimetric survey of OB supergiants is being conducted by Karen Bjorkman (Bjorkman 1994). The observed random PA values suggest that instabilities in an otherwise spherical wind (rather than in a disk) are the cause of the variations. The less luminous Be stars, which show disks, are discussed by K. Bjorkman elsewhere in these proceedings.

Another class of LHS is the Ofpe/WN9 stars, of which ten or so are known in the Magellanic Clouds. They may be O stars in transition to the WR Stage that experience an LBV stage with Ofpe/WN9 characteristics in quiescence (Crowther and Smith 1997). R127 (section 3.1) has actually become an LBV from an Ofpe/WN9 object (Stahl et al. 1983). Pasquali et al. (1997) showed that HDE 269445 has a two component wind. Undoubtedly this class as a whole would be a prime target for polarimetric studies.

\section{Conclusions}

Imaging and spectropolarimetry data indicate that non-spherically symmetric winds about LHS are the norm. In addition to the suggested systematic observations, other new polarimetric techniques, such as using the Hanle effect in the UV for sensitive detection of magnetic fields (Nordsieck and Harris 1996) look promising. In addition, the new generation of large aperture telescopes such as Gemini and VLT will offer polarimetric capabilities that will be important particularly for the study of objects in the Magellanic Clouds. At the same time, detailed envelope modeling is just becoming possible especially due to Monte Carlo techniques, providing an important feedback on theoretical models. The next few years are bound to witness the coming of age of polarimetry of Luminous Hot Stars and the tapping of its full potential.

AMM thanks the SOC for the invitation and acknowledges support from Fapesp (grants 97/11299-2 and 98/04267-0) and CNPq. CVR has received financial support from Fapesp (grants 98/1443-1 and 92/1812-0).

\section{References}

de Araújo, F.X., de Freitas- Pacheco, J.A., Petrini, D. (1994): MNRAS 267, 501 
Bjorkman, K. S. (1994): Ap\&SS 221,335

Brown, J. C., McLean, I. S., Emslie, A. G. (1978): A\&A 68, 415

Clampin, M. et al. (1993): ApJ 410, L35

Clampin, M. et al. (1995): AJ 110, 251

Crowther, P.A., Smith, L.J. (1997): A\&A 320, 500

Drissen, L., Robert, C., Moffat, A. F. J. (1992): ApJ 386, 288

Fliegner, J., Langer, N., Venn, K.A. (1996): A\&A 308, L13

Gummersbach, C.A., Zickgraf, F.-J., Wolf, B. (1995): A\&A 302, 409

Harries, T. J., Hillier, D. J., Howarth, I. D. (1998): MNRAS 296, 1072

Hayes, D. P. (1985): ApJ 289, 726

Ignace, R., Cassinelli, J. P., Bjorkman, J. E. (1996): ApJ 459, 671

Leitherer, C. Zickgraf, F.-J. (1987): A\&A 174, 103

Leitherer, C. et al. (1994): ApJ 428, 292

Lupie, O. L., Nordsieck, K. H. (1987): AJ 93, 214

Maeder, A. (1996): In: Leitherer, C., Fritze-von-Alvensleben, U., Huchra, J. (eds.) From Stars to Galaxies. ASP Conf. Ser. 98, San Francisco, p. 141

Magalhães, A. M. (1992): ApJ 398, 286

Magalhães A. M. et al. (1996): In: Roberge W. G., Whittet D. C. B. (eds.) Polarimetry of the Interstellar Medium. ASP Conf. Ser. 97, San Francisco, p. 118

Melgarejo, R., Magalhães, A.M., Rodrigues, C.V. (1999): in preparation.

Moffat, A.F.J., Robert, C. (1991) In: van der Hucht, K. A., Williams, P. M. (eds.) Wolf-Rayet: Binaries, colliding winds and evolution. Dordrecht, Kluwer, p. 109

Nordsieck, K. H., Harris, W. M. (1996): In: Roberge W. G., Whittet D. C. B. (eds.) Polarimetry of the Interstellar Medium. ASP Conf. Ser. 97, San Francisco, p. 100

Nota, A. et al. (1995): ApJ 448, 788

Pasquali, A. et al. (1997): A\&A 327, 265

Roberge, W.G, Whittet, D.C.B. (1996) (eds.) Polarimetry of the Interstellar Medium. ASP Conf. Series 97, San Francisco.

Robert, C. et al. (1989): ApJ 347, 1034

Robert, C. et al. (1992): ApJ 397, 277

Rodrigues, C. V., Magalhães, A. M. (1995): In: van der Hucht, K. A., Williams, P. M. (eds.) Wolf-Rayet: Binaries, colliding winds and evolution. Dordrecht, Kluwer, p. 260

St.-Louis, N. et al. (1988): ApJ 330, 286

St.-Louis, N. et al. (1993): ApJ 410, 342

Schulte-Ladbeck, R. E. (1997): Rev. Mod. Astron. 10, 135

Schulte-Ladbeck, R. E., Clayton, G. C. (1993): AJ 106, 790

Schulte-Ladbeck, R. E. et al. (1992): ApJ 387, 347

Schulte-Ladbeck, R. E. et al. (1993): ApJ 407, 723

Schulte-Ladbeck, R. E. et al. (1994a): Space Sci. Rev. 66, 193

Schulte-Ladbeck, R. E. et al. (1994b): ApJ 429, 846

Stahl, O. et al. (1983): A\&A 127, 49

Taylor, M. et al. (1991a): AJ 102, 1197

Taylor, M. et al. (1991b): ApJ 382, L85

Weis, K. et al. (1997): A\&A 320, 568

Wood, K. et al. (1996): ApJ 461, 828

Zickgraf, F.-J. et al. (1996a): A\&A 315, 510

Zickgraf, F.-J. et al. (1996b): A\&A 309, 505 


\section{Discussion}

H. Henrichs: What about $\mathrm{O}$ stars?

M. Magalhães: The work of Lupie and Nordsieck (1987) showed that 6 OB stars (out of 8 , mostly supergiants) had intrinsic polarisation. Their polarimetric variability correlated directly with the average $\mathrm{H} \alpha$ equivalent widths and inversely with $v \sin i$. From the detected variability they concluded that the wind structures in the envelopes were short lived and formed at different distances from the stars.

In the on-going spectropolarimetric survey of hot stars at the University of Wisconsin conducted by Karen Bjorkman, the OB supergiants show significant polarisation variability, with 9 out of 18 objects showing the effect in more than one observation. The observed random position angles again basically agree with the above conclusions.

R. Schulte-Ladbeck: I was thinking about how the $\mathrm{B}[\mathrm{e}]$ polarisation might help us learn about disk properties. Currently, observations of the linear polarisation have two problems: firstly, the only spectropolarimetric data set is that of Schulte-Ladbeck \& Clayton. In this case, the $\mathrm{H}_{2}$ line effect allows us to estimate the interstellar polarisation so that we may determine the amount of intrinsic polarisation. All other stars have only broad-band polarimetry and the interstellar polarisation correction is less secure.

Secondly, the only public-user polarimeter on the southern hemisphere is on the AAT. The throughput of the system, unlike HPOL, does not remain high shortward of $4000 \AA$. Therefore no data exists for the polarisation variation across the Balmer discontinuity. One might try in the future to obtain measurements across the Paschen jump.

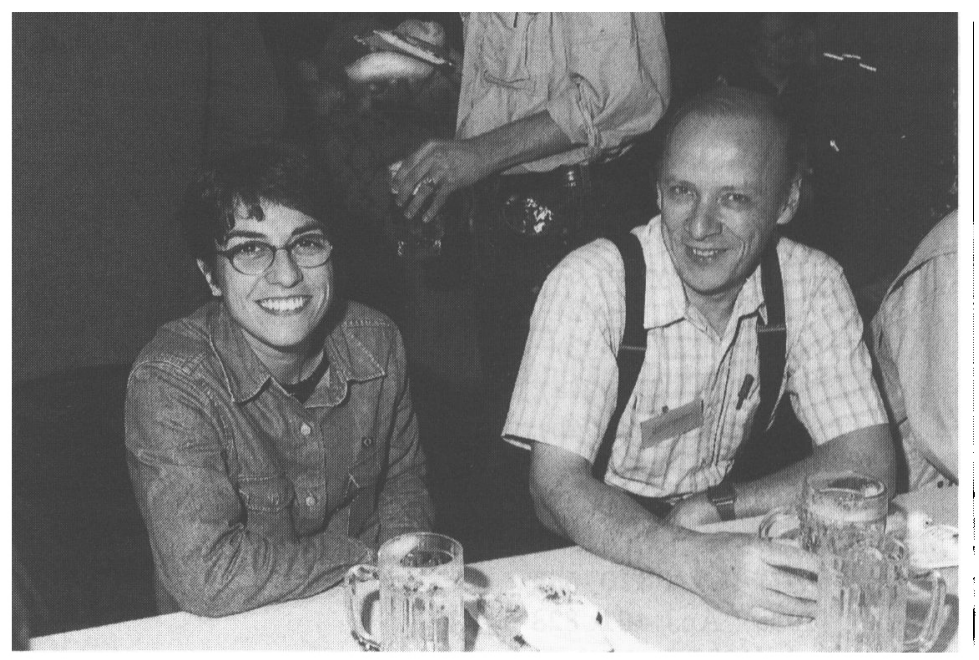

Cláudia Rodrigues and Mário Magalães 